\title{
Particle dark matter searches in the anisotropic sky
}

\section{Nicolao Fornengo * and Marco Regis}

Dipartimento di Fisica, Università di Torino and Istituto Nazionale di Fisica Nucleare, Torino, Italy

\section{Edited by:}

Stefano Morisi, University of

Wurzburg, Germany

\section{Reviewed by:}

Marco Cirelli, Centre National de la

Recherche Scientifique, France

Jihn E. Kim, Kyung Hee University,

South Korea

\section{*Correspondence:}

Nicolao Fornengo, Dipartimento di Fisica, Università di Torino and Istituto Nazionale di Fisica Nucleare, Via P. Giuria 1, Torino 10125, Italy e-mail: fornengo@to.infn.it
Anisotropies in the electromagnetic emission produced by dark matter (DM) annihilation or decay in the extragalactic sky are a recent tool in the quest for a particle DM evidence. We review the formalism to compute the two-point angular power spectrum in the halo-model approach and discuss the features and the relative size of the various auto- and cross-correlation signals that can be envisaged for anisotropy studies. From the side of particle DM signals, we consider the full multi-wavelength spectrum, from the radio emission to X-ray and gamma-ray productions. We discuss the angular power spectra of the auto-correlation of each of these signals and of the cross-correlation between any pair of them. We then extend the search to comprise specific gravitational tracers of DM distribution in the Universe: weak-lensing cosmic shear, large-scale-structure matter distribution and CMB-lensing. We have shown that cross-correlating a multi-wavelength DM signal (which is a direct manifestation of its particle physics nature) with a gravitational tracer (which is a manifestation of the presence of large amounts of unseen matter in the Universe) may offer a promising tool to demonstrate that what we call DM is indeed formed by elementary particles.

Keywords: particle dark matter, anisotropy, multiwavelength signals, gravitational lensing

\section{INTRODUCTION}

The presence of a large amount of dark matter (DM) in the Universe is now supported by means of a numerous and converging astrophysical and cosmological probes. These probes allow to determine that the average, horizon-scale, DM contribution to the energy density of the Universe is six times larger than the baryonic density. In a spatially flat $\Lambda \mathrm{CDM}$ scenario the DM energy density is about $25 \%$ of the critical density of the Universe [1]. On smaller scales, the DM is observed to be distributed quite anisotropically: it forms a hierarchical network of cosmic structures, from the large-scale galaxy clusters down to the small-scale galaxies and their inner parts. This observational picture is consistent with the theoretical understanding of cosmic structure formation through gravitational instability, based on a DM component which is relatively cold. The cold dark matter (CDM) paradigm, with CDM evolving in an expanding Universe is able to reproduce the statistical properties of the large scale structure (LSS) of the Universe, and predicts a galaxy formation pattern which is consistent, at least to a large extent, to observations. Although the common picture of CDM structure formation may be posed under discussion at very small scales, and even though the true role of baryons if the galaxy formation process has only recently started to be investigated, nevertheless the general picture of the LSS formation is robust (for recent reviews on the subject, see, e.g., Ref. [2]).

The most common interpretation of the DM in the Universe relies on the simple and quite natural paradigm of particle excitation in the hot early plasma, with subsequent decoupling and formation of a cosmological abundance of DM particles. CDM is then naturally explained in terms of relic particles which need to be relatively massive (in order to be cold at decoupling) and weakly interacting (such that they freeze-out with the correct relic abundance). Although alternative hypothesis are possible (axions or axion-like particles, non-thermal or non-symmetric DM production) the WIMP mechanism offers a viable and natural solution to the presence of DM in the Universe.

DM evidence is currently of pure gravitational origin: galaxycluster dynamics, rotational curves of spiral galaxies, gravitational lensing observations, hydrodynamical equilibrium of hot gas in galaxy clusters, the same energy budget of the Universe and the theory of structure formation, are all probes based on the gravitational effects induced by the presence of large amounts of DM. However, if DM is composed by elementary particles, vestiges of the early thermal phase, it is expected to produce also nongravitational signals, specifically related to its particle-physics nature. A large number of astrophysical DM signals are currently explored: except for direct detection, which relies on the direct scattering of the DM particles with a low-background detector in an underground laboratory, all other foreseen signals are of indirect origin and rely on the products of annihilation (or decay) of the DM particle in the astrophysical environment, either galactic or extra-galactic. These indirect signals comprise charged galactic exotic cosmic-rays species (electrons/positrons, antiprotons, antideuterons), the whole spectrum of electromagnetic emission (from radio to gamma-rays) and neutrinos.

The indirect detection signals are intrinsically anisotropic, since they are produced by DM structures in the Universe, and structures are present potentially at any scale: galaxy clusters on the largest scales, individual galaxies inside clusters, subhalos inside galaxies. Especially electromagnetic signals, which more directly trace the distribution of the DM that produces them, are expected to exhibit some level of anisotropy. Bright enough 
DM objects (at any given wavelength) would appear as resolved sources: an example could be a radio or gamma-ray halo surrounding and comprising a galaxy cluster or a single galaxy (dwarf galaxies, being DM dominated objects, are interesting targets for this search) or even galactic subhalos which could appear as isolated objects in the radio or gamma-rays sky). Unresolved DM sources, i.e., with brightnesses below the detector sensitivity, produce instead a diffuse flux of photons which contains a subdominant level of anisotropy, due to the sum of a large number of faint sources related to the anisotropic DM distribution. In this case, the effect of anisotropies could be identified on a statistical basis, by means of studying observables related to angular correlations of the spatial map of the signal, the most typical and relevant one being the two-point angular power spectrum. We comment that also the diffuse neutrino signal would be endowed of the anisotropic feature, since neutrinos are necessarily produced in the same processes that lead also to prompt gamma-rays: however, the mechanisms of neutrino detection introduce a larger spatial uncertainty, as compared to photon detectors, and moreover the detection rates for neutrinos are significantly lower than for photons, due to the weak nature of neutrino interactions.

Indirect detection searches of the anisotropic DM sky can follow three directions: the study of anisotropies of a single electromagnetic signal, which represents an auto-correlation observable; the cross-correlation of anisotropies in two different electromagnetic signals; the cross-correlation of an electromagnetic signal with gravitational tracers of the DM distribution in the Universe. The first option has already been attempted both for gammarays [3-11] (also on the data recently reported by the Fermi Collaboration [12]) and for the radio signal [13, 14] (for some discussions in the X-ray band, see Ref. [15]).

Different wavelengths are emitted by DM through different physical mechanisms: gamma-rays may be promptly produced in the DM annihilation/decay event through production of mesons (mostly neutral pions) or baryons, which subsequently decay, or as final-state radiation of charged particles; X-rays (and gamma-rays) are produced by inverse-Compton scattering of the electrons/positrons produced by DM annihilation/decay on the interstellar radiation fields and on the $\mathrm{CMB}$; radio waves are due to synchrotron emission of the same electrons/positrons in the magnetic fields present in the cosmic structure where they are produced. The different electromagnetic signal therefore probe differently the astrophysical properties of the emitting DM structure. Prompt gamma-ray emissions most directly retain the spatial features of the DM density field, while the radiative signals feel also the electrons/positrons spatial diffusion and energy loss, as well as the distribution of magnetic fields. On the other hand, the angular resolution of gamma-rays detectors is lesser than the one accessible to radio (and X-ray) telescopes. Each signal has therefore different features, and the possibility to exploit all types of signal can potentially help in extracting a true DM signal.

Let us notice here that the relative size of the electromagnetic emission at different wavelengths from DM particles, can significantly change depending on the particle DM properties. For example, a DM particle that can annihilate into hadrons, typically produces softer spectra both of gamma-rays and of electrons/positrons, as compared to leptonic annihilations: this reflects on the spectral features of both the prompt and radiative signals, and to some extent also on the spatial features of the radiative signal, since diffusion and energy losses of electrons/positrons depend on energy. Similar arguments apply to the case of decaying DM. The spectral features then depend also on the DM mass, since non-relativistic annihilations (or decays) set an upper bound on the maximal accessible energy at the value of the DM mass (or half the value of the DM mass for DM decay). All these features can then affect differently the relative size also of the auto-correlation signals.

The second possibility, i.e., cross-correlation between different electromagnetic signal, has not yet been studied: by employing the signal coming at two different wavelengths, we can probe in an independent and richer way the different responses of a DM signal to the DM particle properties (as discussed above), and to some extent also to the properties of the DM structures (again, for the reasons discussed above on the way an electromagnetic signal is produced). In the following we will therefore discuss the potentialities for a DM signal in the different cross-correlation channels and try to quantify the mutual impact in the signal extraction. We will explicitly discuss radio-gamma, radio-X and X-gamma cross-correlations.

The third option is potentially the most relevant one, since it attempts to directly correlate two distinctive features of particle DM: an electromagnetic signal, which is a typical and unambiguous manifestation of the DM being an elementary particle, from one side; the direct probe of the existence of DM in the Universe, which is seen through the gravitational effects induced by the presence of DM in the Universe, on the other side. A positive signal in this cross-correlation channel would provide direct evidence that what is measured by means of gravitational probes is indeed due to DM in terms of an elementary particle and is not, e.g., a manifestation of alternative theories of gravity. This third approach has been pioneered in Ref. [16], where the cross-correlation of the gamma-rays emission with the weaklensing cosmic shear has been studied and shown to represent a viable and promising novel channel of investigation. In this paper we extend the cross-correlation studies to two additional gravitational probes: tracers of the LSS and the very recent observation of the CMB lensing signal. We also extend the analyses to comprise not only gamma-rays, but also the radio and $\mathrm{X}$-rays emissions from DM, in order to establish the potentialities of the full range of possibilities.

In this paper we concentrate on the theory of extragalactic DM anisotropies. We discuss the features, sizes and comparisons of the various auto- and cross-correlation signals in the anisotropic sky, for DM annihilations and decays and looking into the whole electromagnetic spectrum, with the aim of exploring the full range of possible signals and correlations, and of setting the ground for future analyses. First, we will carefully outline the formalism needed to perform computations of extragalactic DM anisotropies. We then review some channels already discussed in the literature (gamma-rays autocorrelations, radio autocorrelations and gamma rays/cosmic shear cross-correlation), and extend the range of investigation to propose new channels: for the signals related to the particle physics nature of DM, we explicitly discuss the auto- and mutual cross-correlations in the 
whole electromagnetic spectrum, by adding to the study the gamma/radio case and the auto- and cross-correlation signals involving X-rays; for the signals able to correlate the gravitational probes of DM in the Universe to the particle-physics ability to produce photon fluxes, we extend the idea to investigate the gamma-rays/cosmic-shear correlation, originally proposed in Ref. [16], to further adopt radio and X-rays emissions from one side, and to embrace large-scale-structure tracers [17] and CMB lensing from the other side. A second step of this complex type of analysis will be a detailed study of the different, various and intricate astrophysical emitters at the different wavelengths (like active galactic nuclei, star forming galaxies, etc.) which also contribute to the anisotropic sky and represent the background over which the signal we are discussing have to be confronted, as well as an accurate assessment of the observational capabilities. We leave these parts of the analysis to future works.

The paper starts with a review of the general formalism apt to derive the two-point angular power spectrum of correlation signals, and details the steps relevant for studying both DM annihilation (which depends on the square of the DM density field) and DM decay (which instead depends linearly on the DM density) cases. Then discusses the features of the relevant ingredients which enter the correlation signal: the three-dimensional power spectrum, both for the auto- and cross-correlation, and the window functions for each electromagnetic and gravitational signal. Angular power spectrum predictions are then shown and discussed for all types of correlations signals: auto correlations in the radio, $\mathrm{X}$ and gamma-rays bands; cross-correlations among them; cross-correlations between the radio or gamma-rays emission and the gravitational probes (cosmic shear, CMB lensing, LSS observables). Results will be presented for benchmark cases and for both annihilating and decaying DM.

\section{GENERAL FORMALISM}

In this section we review the general formalism to derive the angular power spectrum of the auto- and cross-correlation of any given pair of intensity fields, which in our analysis are given by the radio, X-ray, gamma-ray DM fluxes, or the cosmic shear, LSS, CMB-lensing maps.

The source intensity $I_{g}$ along a given direction $\vec{n}$ can be written as:

$$
I_{g}(\vec{n})=\int d \chi g(\chi, \vec{n}) \tilde{W}(\chi)
$$

where $\chi(z)$ denotes the radial comoving distance (function of the redshift $z), g(\chi, \vec{n})$ is the density field of the source, and $\tilde{W}(\mathrm{x})$ is the window function (which is function of the distance but does not depend on the specific direction $\vec{n}$ ). We can also define a normalized version of the window function as $W(\chi)=$ $\langle g\rangle \tilde{W}(\chi)$, such that the average intensity is obtained by integration of the normalized window function along the distance: $\left\langle I_{g}\right\rangle=\int d \chi W(\chi)$.

The window function brings the information on how the actual observable related of the signal under study is distributed in redshift. It will act as a weight for the different redshift contributions of the 3D power spectrum when computing the angular power spectrum. Its specific form varies depending on whether we are studying the cosmological emission of an electromagnetic signal from DM annihilation (or decay), or instead the images of background galaxies (which are lensed by the structures) in the case of cosmic shear, or the CMB flux at the large scattering surface in the case of CMB lensing, or the distribution of astrophysical sources and of their light emission (typically in the visible, infrared or radio wavelength) in the case of LSS tracers. The specific form of $W(x)$ for all the cases under study will be reported in a dedicated section below.

By defining the intensity fluctuation as $\delta I_{g}(\vec{n}) \equiv I_{g}(\vec{n})-\left\langle I_{g}\right\rangle$ and expanding the fluctuation field in terms of spherical harmonics, $\delta I_{g}(\vec{n})=\left\langle I_{g}\right\rangle \sum_{\ell m} a_{\ell m} Y_{\ell m}(\vec{n})$, the (dimensionless) $a_{\ell m}$ coefficients can be expressed as:

$$
\begin{aligned}
a_{\ell m} & =\frac{1}{\left\langle I_{g}\right\rangle} \int d \vec{n} \delta I_{g}(\vec{n}) Y_{\ell m}^{*}(\vec{n}) \\
& =\frac{1}{\left\langle I_{g}\right\rangle} \int d \vec{n} d \chi f_{g}(\chi, r) W(\chi) Y_{\ell m}^{*}(\vec{n})
\end{aligned}
$$

where the orthonormal relations for $Y_{\ell m}$ have been used and where $f_{g} \equiv g /\langle g\rangle-1$. A spatial Fourier transformation of $f_{g}$ and the use of the Rayleigh expansion of a plane wave into spherical harmonics and spherical Bessel functions $j_{\ell}$, allows to derive:

$$
\begin{aligned}
a_{\ell m}= & \frac{1}{\left\langle I_{g}\right\rangle} \int d \vec{n} d \chi \frac{d \boldsymbol{k}}{(2 \pi)^{3}} \hat{f}_{g}(\chi, \boldsymbol{k}) e^{i \boldsymbol{k} \cdot \boldsymbol{r}} W(\chi) Y_{\ell m}^{*}(\vec{n}) \\
= & \frac{1}{\left\langle I_{g}\right\rangle} \int d \vec{n} d \chi \frac{d \boldsymbol{k}}{2 \pi^{2}} \hat{f}_{g}(\chi, \boldsymbol{k}) \\
& {\left[\sum_{\ell^{\prime} m^{\prime}} i^{\ell^{\prime}} j_{\ell^{\prime}}(k \chi) Y_{\ell^{\prime} m^{\prime}}^{*}(\hat{\boldsymbol{k}}) Y_{\ell^{\prime} m^{\prime}}(\vec{n})\right] W(\chi) Y_{\ell m}^{*}(\vec{n}) } \\
= & \frac{i^{\ell}}{\left\langle I_{g}\right\rangle} \int d \chi W(\chi) \int \frac{d \boldsymbol{k}}{2 \pi^{2}} \hat{f}_{g}(\chi, \boldsymbol{k}) j_{\ell}(k \chi) Y_{\ell m}^{*}(\hat{\boldsymbol{k}})
\end{aligned}
$$

where we used $\vec{r}=\chi \vec{n}$.

The angular power spectrum (PS) is defined as $C_{\ell}^{(i j)}=$ $\left\langle\sum_{m} a_{\ell m}^{(i)} a_{\ell m}^{(j) *}\right\rangle$, where $i, j=1,2$ label the two signals for which the correlation is studied (e.g., $i=$ gamma-rays and $j=$ cosmicshear), and the brackets $\langle\ldots\rangle$ denote ensemble average. Clearly, the cross-correlation angular PS $C_{\ell}^{(i j)}=C_{\ell}^{(j i)}$, as can be easily derived from the relation $a_{\ell}=(-1)^{m} a_{\ell-m}^{*}$, while autocorrelation occurs for $i=j$. By using Equation (3), the correlation angular PS takes the form:

$$
\begin{aligned}
C_{\ell}^{(i j)}= & \frac{1}{\left\langle I_{i}\right\rangle\left\langle I_{j}\right\rangle} \int d \chi W_{i}(\chi) \int d \chi^{\prime} W_{j}\left(\chi^{\prime}\right) \int \frac{d \boldsymbol{k}}{2 \pi^{2}} \\
& \int \frac{d \boldsymbol{k}^{\prime}}{2 \pi^{2}}\left\langle\hat{f}_{g_{i}}(\chi, \boldsymbol{k}) \hat{f}_{g_{j}}^{*}\left(\chi^{\prime}, \boldsymbol{k}^{\prime}\right)\right\rangle j_{\ell}(k r) j_{\ell^{\prime}}\left(k^{\prime} r^{\prime}\right) Y_{\ell m}(\hat{\boldsymbol{k}}) Y_{\ell^{\prime} m^{\prime}}^{*}\left(\hat{\boldsymbol{k}}^{\prime}\right) \\
= & \frac{2}{\pi\left\langle I_{i}\right\rangle\left\langle I_{j}\right\rangle} \int d \chi W_{i}(\chi) \int d \chi^{\prime} W_{j}\left(\chi^{\prime}\right) \\
& \int d \boldsymbol{k} P_{i j}\left(k, \chi, \chi^{\prime}\right) j_{\ell}(k r) j_{\ell^{\prime}}\left(k r^{\prime}\right) Y_{\ell m}(\hat{\boldsymbol{k}}) Y_{\ell^{\prime} m^{\prime}}^{*}(\hat{\boldsymbol{k}})
\end{aligned}
$$




$$
\begin{aligned}
= & \frac{2}{\pi\left\langle I_{i}\right\rangle\left\langle I_{j}\right\rangle} \int d \chi W_{i}(\chi) \\
& \int d \chi^{\prime} d k k^{2} W_{j}\left(\chi^{\prime}\right) P_{i j}\left(k, \chi, \chi^{\prime}\right) j_{\ell}(k r) j_{\ell}\left(k r^{\prime}\right) \\
= & \frac{1}{\left\langle I_{i}\right\rangle\left\langle I_{j}\right\rangle} \int \frac{d \chi}{\chi^{2}} W_{i}(\chi) W_{j}(\chi) P_{i j}(k=\ell / \chi, \chi)
\end{aligned}
$$

where in the second step we introduced the definition of the three-dimensional (3D) power-spectrum $P_{i j}$ through $\left\langle\hat{f}_{g_{i}}(\chi, \boldsymbol{k}) \hat{f}_{g_{j}}^{*}\left(\chi^{\prime}, \boldsymbol{k}^{\prime}\right)\right\rangle=(2 \pi)^{3} \delta^{3}\left(\boldsymbol{k}-\boldsymbol{k}^{\prime}\right) P_{i j}\left(k, \chi, \chi^{\prime}\right)$, and in the last step we assumed the Limber approximation [18-20] to hold for such PS.

The next step is to compute the explicit form for the specific 3D power spectra $P_{i j}$, which are the Fourier transform of the twopoint correlation functions (2PCF) in real space: $\xi_{i j}^{(2)}(\boldsymbol{x}, \boldsymbol{y}) \equiv$ $\left\langle f_{g_{i}}(\boldsymbol{x}) f_{g_{j}}(\boldsymbol{y})\right\rangle$. Following Ref. [21], we assume that the density field can be expressed as the sum of independent seeds (i.e., of discrete masses, in the case of the gravitational tracers, or the ensuing electromagnetic emission):

$$
\begin{aligned}
f(\boldsymbol{x})= & \sum_{a} f\left(m_{a}, \boldsymbol{x}-\boldsymbol{x}_{a}\right)=\int d m \int d^{3} \boldsymbol{x}^{\prime} \\
& \sum_{a} \delta^{3}\left(\boldsymbol{x}^{\prime}-\boldsymbol{x}_{a}\right) \delta\left(m-m_{a}\right) f\left(m, \boldsymbol{x}-\boldsymbol{x}^{\prime}\right)
\end{aligned}
$$

where $a$ labels the seeds, the $\delta$ 's are Dirac-delta functions, and we take the mass $m$ to be the parameter which characterizes the seeds. The seed density can be expressed as: $d n / d m=$ $\left\langle\sum_{a} \delta^{3}\left(\boldsymbol{x}-\boldsymbol{x}_{a}\right) \delta\left(m-m_{a}\right)\right\rangle$, where $\langle\ldots\rangle$ denotes the ensemble average over of all possible seed distributions. With these definitions, the $2 \mathrm{PCF}$ reads:

$$
\begin{aligned}
\xi^{(2)}(\boldsymbol{x}, \boldsymbol{y})= & \int d m_{1} d m_{2} d^{3} \boldsymbol{x}_{1} d^{3} \boldsymbol{x}_{2}\left\langle\sum_{a} \delta^{3}\left(\boldsymbol{x}_{1}-\boldsymbol{x}_{a}\right) \delta\left(m_{1}-m_{a}\right)\right. \\
& \left.\sum_{b} \delta^{3}\left(\boldsymbol{x}_{2}-\boldsymbol{x}_{b}\right) \delta\left(m_{2}-m_{b}\right)\right\rangle f_{1}\left(m_{1}, \boldsymbol{x}-\boldsymbol{x}_{1}\right) \\
& f_{2}\left(m_{2}, \boldsymbol{y}-\boldsymbol{x}_{2}\right)
\end{aligned}
$$

The correlation of a seed $a$ with mass $m_{1}$ at position $x_{1}$ with a different seed $b$ with mass $m_{2}$ at position $x_{2}$ is provided by the seed-2PCF $\xi_{s}^{(2)}\left(m_{1}, m_{2}, \boldsymbol{x}_{1}, \boldsymbol{x}_{2}\right)$, and it is not difficult to see that:

$$
\begin{aligned}
\left\langle\sum_{a} \delta^{3}\left(\boldsymbol{x}_{1}-\boldsymbol{x}_{a}\right) \delta\left(m_{1}-m_{a}\right) \sum_{b} \delta^{3}\left(\boldsymbol{x}_{2}-\boldsymbol{x}_{b}\right) \delta\left(m_{2}-m_{b}\right)\right\rangle \\
=\frac{d n}{d m_{1}} \frac{d n}{d m_{2}}\left[1+\xi_{s}^{(2)}\left(m_{1}, m_{2}, \boldsymbol{x}_{1}, \boldsymbol{x}_{2}\right)\right] \\
\quad+\frac{d n}{d m_{1}} \delta^{3}\left(\boldsymbol{x}_{1}-\boldsymbol{x}_{2}\right) \delta\left(m_{1}-m_{2}\right)
\end{aligned}
$$

which leads to:

$$
\begin{aligned}
\xi^{(2)}(\boldsymbol{x}, \boldsymbol{y})= & \int d m d^{3} \boldsymbol{x}_{1} \frac{d n}{d m} f_{1}\left(\boldsymbol{x}-\boldsymbol{x}_{1}, m\right) f_{2}\left(\boldsymbol{y}-\boldsymbol{x}_{1}, m\right) \\
& +\int d m_{1} d m_{2} d^{3} \boldsymbol{x}_{1} d^{3} \boldsymbol{x}_{2} \frac{d n}{d m_{1}} \frac{d n}{d m_{2}} f_{1}\left(\boldsymbol{x}-\boldsymbol{x}_{1}, m_{1}\right) \\
& f_{2}\left(\boldsymbol{y}-\boldsymbol{x}_{2}, m_{2}\right) \xi_{s}^{(2)}\left(m_{1}, m_{2}, \boldsymbol{x}_{1}, \boldsymbol{x}_{2}\right)
\end{aligned}
$$

Coming back to the fact that the $3 \mathrm{D}$ power spectrum $P_{i j}$ is the Fourier transform of $\xi^{(2)}(\boldsymbol{x}, \boldsymbol{y})$, and writing $f_{i}$ in terms of their Fourier transforms $\hat{f}_{i}$, one obtains:

$$
\begin{aligned}
P_{i j}(k)= & \int d m \frac{d n}{d m} \hat{f}_{i}^{*}(k \mid m) \hat{f}_{j}(k \mid m) \\
& +\int d m_{1} d m_{2} \frac{d n}{d m_{1}} \frac{d n}{d m_{2}} \hat{f}_{i}^{*}\left(k \mid m_{1}\right) \hat{f}_{j}\left(k \mid m_{2}\right) \\
& P^{(s)}\left(k, m_{1}, m_{2}\right)
\end{aligned}
$$

where the power spectrum of the seed distribution $P^{(s)}$ is the Fourier transform of $\xi_{s}^{(2)}\left(m_{1}, m_{2}, \boldsymbol{x}_{1}, \boldsymbol{x}_{2}\right)$.

In our analysis, we often refer to mass density fluctuations. In this case, the seed-2PCF is the (homogeneous and isotropic) linear correlation function of matter (notice that it has to be the linear one, since in Equation (6) we wrote the density field as a linear superposition of seeds), $\xi_{s}^{(2)}\left(m_{1}, m_{2}, \boldsymbol{x}_{1}, \boldsymbol{x}_{2}\right)=\xi_{\text {lin }}^{(2)}\left(\left|\boldsymbol{x}_{i}-\boldsymbol{x}_{j}\right|\right)$. The corresponding power spectrum is the customary $P^{\text {lin }}(k)$. For other objects considered in the following, i.e., DM halos and astrophysical sources, we will assume (as usually done) that their $2 \mathrm{PCF} \xi_{s}^{(2)}$ can be related to the linear correlation function of matter by means of $\xi_{s, i j}^{(2)}\left(m_{1}, m_{2}, \boldsymbol{x}_{i}, \boldsymbol{x}_{j}\right) \approx b_{i}\left(m_{1}\right) b_{j}\left(m_{2}\right) \xi_{\text {lin }}^{(2)}\left(\left|\boldsymbol{x}_{i}-\boldsymbol{x}_{j}\right|\right)$ where $b_{i}(m)$ is the linear bias between the object $i$ and matter. Thus using $P^{(s)}\left(k, m_{1}, m_{2}\right)=b_{i}\left(m_{1}\right) b_{j}\left(m_{2}\right) P^{\text {lin }}(k)$, we finally arrive at the decomposition of the $3 \mathrm{D}$ power-spectrum into the one-halo and two-halo terms:

$$
P_{i j}(k)=P_{i j}^{1 h}(k)+P_{i j}^{2 h}(k)
$$

where:

$$
\begin{aligned}
P_{i j}^{1 h}(k)= & \int d m \frac{d n}{d m} \hat{f}_{i}^{*}(k \mid m) \hat{f}_{j}(k \mid m) \\
P_{i j}^{2 h}(k)= & {\left[\int d m_{1} \frac{d n}{d m_{1}} b_{i}\left(m_{1}\right) \hat{f}_{i}^{*}\left(k \mid m_{1}\right)\right] } \\
& {\left[\int d m_{2} \frac{d n}{d m_{2}} b_{j}\left(m_{2}\right) \hat{f}_{j}\left(k \mid m_{2}\right)\right] P^{\operatorname{lin}(k)} }
\end{aligned}
$$

Notice that the average $\langle g\rangle$ of the density field of the source is given by:

$$
\bar{g}(z)=\langle g(z, \vec{n})\rangle=\int d m \frac{d n}{d m} \int d^{3} \boldsymbol{x} g(\boldsymbol{x} \mid m, z),
$$

which implies that at small $k$ (where $\hat{f} \sim \int d^{3} \boldsymbol{x} g(\boldsymbol{x} \mid m) / \bar{g}$ ) the terms in the square-brackets in Equation (12) are of order 1 
(except in the case of a significant bias). The 2-halo term is thus normalized to the standard linear matter PS at small $k$, which motivates the normalization of the window function introduced above.

In principle, instead of adopting the above halo-model formalism, one could directly take the results from N-body simulations, generate the corresponding maps of the emissions and then extract the angular power spectrum. On the other hand, the small scales are currently not covered by simulations and in order to make a realistic prediction of the signal (in particular in the annihilating DM scenario) one has to introduce some prescription to include them. Results based on simulations only (without the introduction of small scales) can be significantly biased. The halo-model approach is instead based on tuning three-functions (describing the number of halos $d n / d m$, the mass concentration of halos $c_{\text {vir }}$, and the halo profile $\rho$ ) to the simulation results, where they exist, and defining some appropriate extrapolations in the unsampled regime. The computation is therefore quite neat once those functions have been drawn, and allows for an easier physical insight. Since it is probably unlikely that N-body simulations will cover scales corresponding to the WIMP free-streaming mass in the forthcoming years, such a phenomenological approach will remain a prime way to compute the WIMP angular PS.

\section{THREE-DIMENSIONAL POWER SPECTRA}

The next step is to explicitly derive the three-dimensional power spectra, defined in Equation (10), for the different cases of autocorrelation and cross-correlation involving particle DM signals. This requires to adopt, for each specific case, the appropriate intensity-field function $g$.

We will only consider a DM source with a spatial profile directly tracing the DM density profile (or its square in the annihilating scenario). This is typically the case for prompt emission of gamma-rays, while for the radio emission, and for the X-ray or gamma-ray signal produced by inverse Compton processes, the radiative emissions associated to electrons and positrons injected by DM can have a different shape either because of the spatial profile of the fields of interaction (the magnetic field for the synchrotron emission which produces the radio signal; the radiation fields, like CMB or starlight, which are responsible for the X-ray, or also gamma-rays, production through inverse Compton scattering; both fields contribute to energy losses), or because of the diffusion of $e^{+} / e^{-}$before emission. In this case the density field of the emission is no longer proportional to the DM density (or its square) and has to be accordingly modified. This modification typically affects only anisotropies on very small scales (see the full formalism in Ref. [14]) and, for simplicity, will be neglected here except for some approximate estimate in the case of the radio emission.

\section{AUTO-CORRELATION}

In the cases of lensing and decaying DM, the density field of the source is directly given by the density distribution $\rho(\boldsymbol{x}): g(\boldsymbol{x})=$ $\rho(\boldsymbol{x})$, and therefore $f(\boldsymbol{x})=\delta(\boldsymbol{x})$, where the $\delta(\boldsymbol{x})$ is the density contrast. This is obvious for decaying DM, while in the lensing case it stems from the fact that gravitational lensing is due to the potential wells of the LSS, which are related to the matter distribution $\rho$ by the Poisson equation. Therefore, from Equations (11) and (12), the non-linear matter PS $P_{\delta \delta}$ is obtained by summing up the following one-halo and two-halo terms:

$$
\begin{aligned}
& P_{\delta \delta}^{1 h}(k)=\int_{m_{\min }}^{m_{\max }} d m \frac{d n}{d m} \tilde{v}(k \mid m)^{2} \\
& P_{\delta \delta}^{2 h}(k)=\left[\int_{m_{\min }}^{m_{\max }} d m \frac{d n}{d m} b_{h}(m) \tilde{v}(k \mid m)\right]^{2} P^{\operatorname{lin}}(k)
\end{aligned}
$$

where $\tilde{v}(k \mid m)$ is the Fourier transform of $\rho(\boldsymbol{x} \mid m) / \bar{\rho}$. In the rest of the paper, we will adopt the halo mass function $d n / d m$ of Ref. [22], the halo concentration from Ref. [23] down to $10^{10} M_{\odot}$ and extrapolating $\mathcal{c}_{\mathrm{vir}}$ at smaller masses following Ref. [24], and a NFW halo density profile [25]. The halo bias $b_{h}$ is taken from Ref. [26]. We compare our halo-model-based predictions for the matter PS with latest results from high-resolution $N$-body simulations [27] in Figure 1. A very good agreement is achieved at low redshift, which is the most relevant epoch for DM purposes, as we will see in the following.

For astrophysical sources (as, e.g., the LSS tracer considered below), which are better characterized by their luminosity $\mathcal{L}$ rather than the mass, the formalism described above can still be adopted by simply replacing the mass function $d n / d m$ with the luminosity function $d n / d \mathcal{L} \equiv \Phi(\mathcal{L}, z)$. Approximating astrophysical sources as point sources, we have $g_{S}\left(\mathcal{L}, \boldsymbol{x}-\boldsymbol{x}^{\prime}\right)=$ $\mathcal{L} \delta^{3}\left(\boldsymbol{x}-\boldsymbol{x}^{\prime}\right)$, and Equations (11) and (12) give:

$$
\begin{aligned}
& P_{S S}^{1 h}(k, z)=\int_{\mathcal{L}_{\min }(z)}^{\mathcal{L}_{\max }(z)} d \mathcal{L} \Phi(\mathcal{L}, z)\left(\frac{\mathcal{L}}{\left\langle g_{S}\right\rangle}\right)^{2} \\
& P_{S S}^{2 h}(k, z)=\left[\int_{\mathcal{L}_{\min }(z)}^{\mathcal{L}_{\max }(z)} d \mathcal{L} \Phi(\mathcal{L}, z) b_{S}(\mathcal{L}, z) \frac{\mathcal{L}}{\left\langle g_{S}\right\rangle}\right]^{2} P^{\operatorname{lin}}(k, z) .
\end{aligned}
$$

Since the term in the square-brackets does not depend on $k$ (because of the point-source approximation) and Equation (13) is now $\left\langle g_{S}\right\rangle=\int_{\mathcal{L}_{\min }(z)}^{\mathcal{L}_{\max }(z)} d \mathcal{L} \Phi \mathcal{L}$, the two-halo term is just a rescaled version of $P^{\text {lin }}$ with the rescaling factor due to the bias (so possibly varying with $z$ ). The one-halo term is constant in $k$, namely it is a "Poisson-noise" term. The power spectrum of LSS tracers can be thus computed starting from the luminosity function of the population under investigation as described above (for gammaray emitters see, e.g., Ref. [16]). However, for a given survey might be not so easy to separate different populations (or impossible when dealing with unresolved contributions, unless using theoretical arguments). To keep the discussion general, here we adopt a common description for all types of galaxies, using a halo occupation distribution of galaxies $N_{\text {gal }}$ and weighting the contributions of different redshifts through the redshift distribution function $d n_{\text {gal }} / d z$. This means that the density field of galaxies can be expressed as $g(\boldsymbol{x}, M, z)=\rho(\boldsymbol{x}, M, z)\left\langle N_{\mathrm{gal}}(M, z)\right\rangle / \bar{n}_{\mathrm{gal}}(z)$, where $\bar{n}_{\text {gal }}=\int d M d n / d M\left\langle N_{\text {gal }}\right\rangle$ and $\rho$ is the host-halo density profile. The expression of $N_{\text {gal }}$ is taken from Ref. [28]. In this 


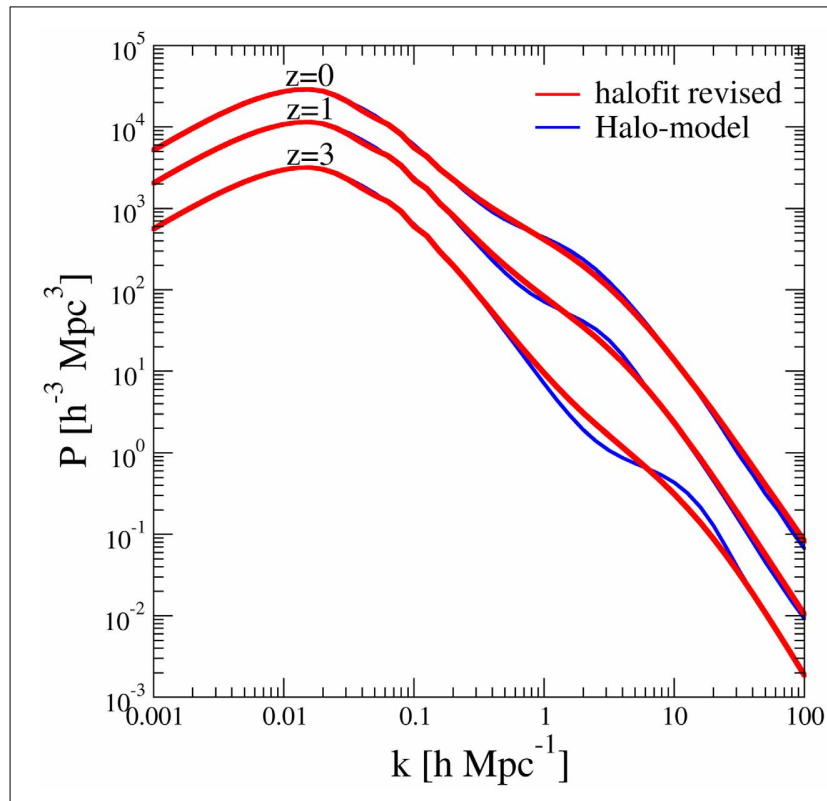

FIGURE 1 | 3D power spectra. Comparison between the non-linear 3D matter-PS obtained with the halo-model considered in this paper and the revised halofit results from high-resolution $\mathrm{N}$-body simulations presented in Ref. [27], at different redshifts.

approximated scenario, we have:

$$
\begin{aligned}
& P_{\text {gal,gal }}^{1 h}(k)=\int_{m_{\min }}^{m_{\max }} d m \frac{d n}{d m} \frac{\left\langle N_{\mathrm{gal}}\left(N_{\mathrm{gal}}-1\right)\right\rangle}{\bar{n}_{\mathrm{gal}}^{2}} \tilde{v}(k \mid m)^{2} \\
& P_{\text {gal,gal }}^{2 h}(k)=\left[\int_{m_{\min }}^{m_{\max }} d m \frac{d n}{d m} b_{h}(m) \frac{\left\langle N_{\mathrm{gal}}\right\rangle}{\bar{n}_{\mathrm{gal}}} \tilde{v}(k \mid m)\right]^{2} P^{\operatorname{lin}}(k) .
\end{aligned}
$$

In the case of annihilating DM, the signal scales with the square of the density field: $g(\boldsymbol{x})=\rho^{2}(\boldsymbol{x})$. In order to derive the associated angular PS, we can use the formalism outlined in the previous section where now we square the expression given in Equation (6) (also here $f(\boldsymbol{x})=\rho(\boldsymbol{x})$, as in the decaying DM case). The correlation function would then lead to a term proportional to:

$$
\begin{gathered}
\left\langle\sum_{i} \delta^{3}\left(\boldsymbol{x}_{1}-\boldsymbol{x}_{i}\right) \delta\left(m_{1}-m_{i}\right) \sum_{j} \delta^{3}\left(\boldsymbol{x}_{2}-\boldsymbol{x}_{j}\right) \delta\left(m_{2}-m_{j}\right)\right. \\
\left.\sum_{k} \delta^{3}\left(\boldsymbol{x}_{3}-\boldsymbol{x}_{k}\right) \delta\left(m_{3}-m_{k}\right) \sum_{l} \delta^{3}\left(\boldsymbol{x}_{4}-\boldsymbol{x}_{l}\right) \delta\left(m_{4}-m_{l}\right)\right\rangle
\end{gathered}
$$

which in turn can be expanded into four-, three-, and two-point seed correlation functions. However, since DM halos (described by $\rho(\boldsymbol{x} \mid m)$ ) are mutually exclusive, an annihilation can only occur if the two particles are within the same halo. This implies that all the terms other than the one- and two-halo contributions (which are the same appearing in Equation 8) vanish.
Therefore, Equation (9) does not formally change, with $f_{a}$ now being $\rho(\boldsymbol{x} \mid m)^{2} /\left\langle\rho^{2}\right\rangle$. The auto-correlation PS $P_{\delta^{2} \delta^{2}}$ is thus given by the sum of the two following terms:

$$
\begin{aligned}
& P_{\delta^{2} \delta^{2}}^{1 h}(k)=\int_{m_{\min }}^{m_{\max }} d m \frac{d n}{d m}\left(\frac{\tilde{u}(k \mid m)}{\Delta^{2}}\right)^{2} \\
& P_{\delta^{2} \delta^{2}}^{2 h}(k)=\left[\int_{m_{\min }}^{m_{\max }} d m \frac{d n}{d m} b_{h}(m) \frac{\tilde{u}(k \mid m)}{\Delta^{2}}\right]^{2} P^{\operatorname{lin}}(k)
\end{aligned}
$$

where $\tilde{u}(k \mid m)$ is the Fourier transform of $\rho^{2}(\boldsymbol{x} \mid m) / \bar{\rho}^{2}$. Our results are only very mildly dependent on the upper mass cutoff (which we set to be $m_{\max }=10^{18} M_{\odot}$ ) and the clustering on large scales has been well sampled by means of simulations and observations. A crucial and uncertain ingredients is instead the DM clustering at very small masses (minimum halo mass $m_{\mathrm{min}}$, concentration parameter, and substructure scheme). Concerning substructures, we include them by adopting the scheme described in Ref. [29], namely by replacing $\rho^{2}(\boldsymbol{x}, m, z)$ with $B(\boldsymbol{x}, m, z) \rho^{2}(\boldsymbol{x}, m, z)$, where $B(\boldsymbol{x}, m, z)$ is a boost function associated to subhalos which multiplies the smooth halo density profile.

In the left panel of Figure 2, we show three specific examples of $3 \mathrm{D}$ power spectra, which refer to DM models endowed with different features: two models with $m_{\min }=10^{-6} M_{\odot}$ (which is the typical WIMP free-streaming mass) with substructures described by adapting the boost-function $B$ to reproduce the Via Lactea (VL) simulation [29] or the Virgo Collaboration (VC) results [8]); one model with $m_{\min }=10^{7} M_{\odot}$ (which is the minimum halo mass currently inferred from dynamical measurements) without substructures. Having normalized the power spectra to be (roughly) equal on large scales, the effect of substructures mainly reflects into boosting the power on Mpc-scales. In the VC scenario the signal from largest halos is indeed strongly boosted by the contribution of subhalos, while in the VL scenario the effect is much milder.

\section{CROSS-CORRELATION}

In the case of cross-correlations of electromagnetic DM signals among themselves, the situation is similar to the one discussed in the previous section for auto-correlations: for decaying DM, since all signals depend linearly on the DM density, the relevant matter PS is $P_{\delta \delta}$, whose one- and two-halo terms are given by Equations $(14,15)$, while for annihilating DM the matter PS is $P_{\delta^{2} \delta^{2}}$, with its component given by Equations $(21,22)$.

In the case of cross-correlation between lensing (which depends linearly on the DM density) and an electromagnetic signal produced by a decaying DM particle, we are again concerned with the $P_{\delta \delta}$ power spectrum, expressed through Equations $(14,15)$.

The case of cross-correlation of astrophysical sources with decaying DM is analogous to the case involving astrophysical sources and lensing signal, both given by:

$$
P_{S \delta}^{1 h}(k, z)=\int_{\mathcal{L}_{\min }(z)}^{\mathcal{L}_{\max }(z)} d \mathcal{L} \Phi(\mathcal{L}, z) \frac{\mathcal{L}}{\left\langle g_{S}\right\rangle} \tilde{v}(k \mid m(\mathcal{L}))
$$



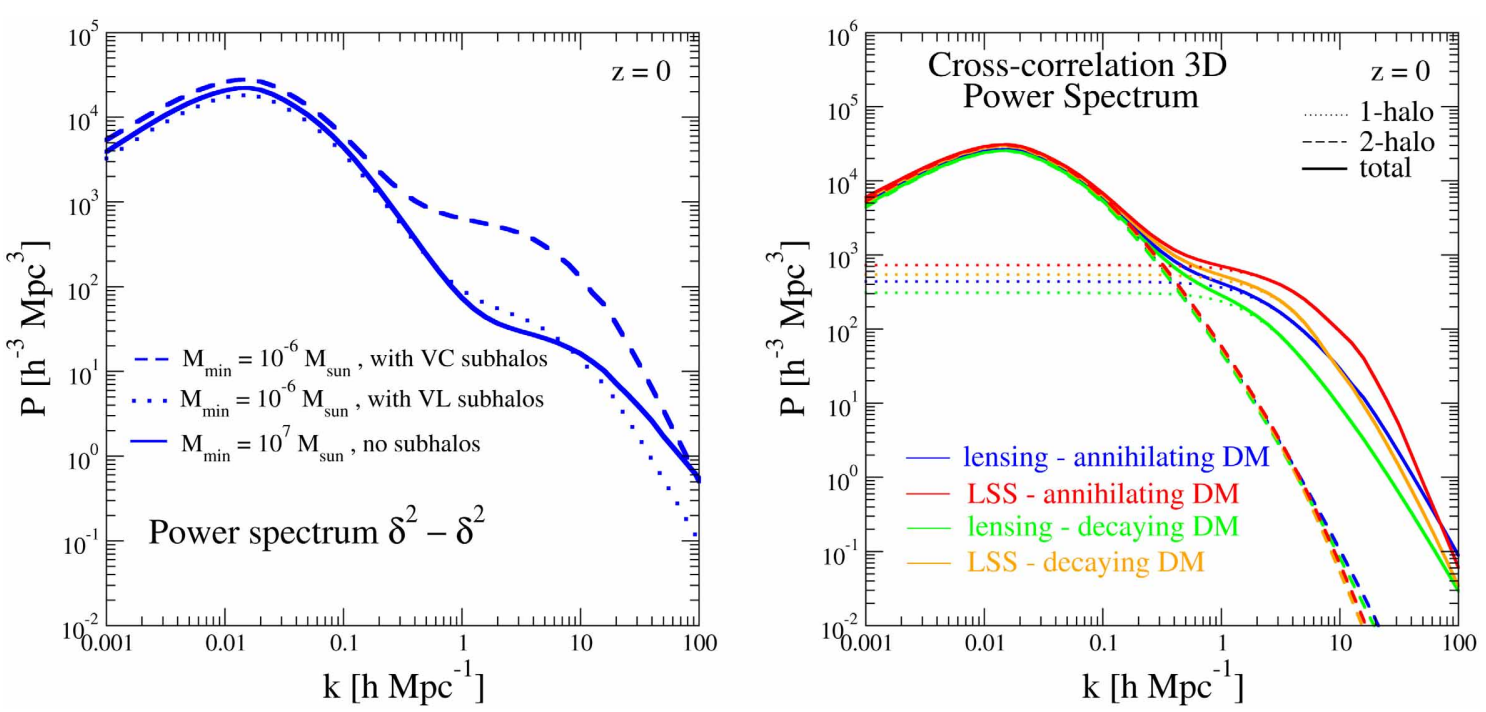

FIGURE 2 | 3D power spectra. 3D power spectra. Left: Auto-correlation 3D PS of annihilating DM in the three scenarios described in the text, at $z=0$. Right: Cross-correlation 3D PS of annihilating/decaying DM with lensing and galaxy tracers; the total, 1-halo and 2-halo terms are explicitly shown.

$$
\begin{aligned}
P_{S \delta}^{2 h}(k, z)= & {\left[\int_{\mathcal{L}_{\min }(z)}^{\mathcal{L}_{\max }(z)} d \mathcal{L} \Phi(\mathcal{L}, z) b_{S}(\mathcal{L}, z) \frac{\mathcal{L}}{\left\langle g_{S}\right\rangle}\right] } \\
& {\left[\int_{m_{\min }}^{m_{\max }} d m \frac{d n}{d m} b_{h}(m) \tilde{v}(k \mid m)\right] P^{\operatorname{lin}}(k, z) . }
\end{aligned}
$$

Notice that this requires a relation between the source luminosity $\mathcal{L}$ and the host-halo mass $m$. In the approximate description introduced above, we have instead:

$$
\begin{aligned}
P_{\text {gal }, \delta}^{1 h}(k)= & \int_{m_{\min }}^{m_{\max }} d m \frac{d n}{d m} \frac{\left\langle N_{\mathrm{gal}}\right\rangle}{\bar{n}_{\mathrm{gal}}} \tilde{v}(k \mid m)^{2} \\
P_{\mathrm{gal}, \delta}^{2 h}(k)= & {\left[\int_{m_{\min }}^{m_{\max }} d m \frac{d n}{d m} b_{h}(m) \frac{\left\langle N_{\mathrm{gal}}\right\rangle}{\bar{n}_{\mathrm{gal}}} \tilde{v}(k \mid m)\right] } \\
& {\left[\int_{m_{\min }}^{m_{\max }} d m \frac{d n}{d m} b_{h}(m) \tilde{v}(k \mid m)\right] P^{\operatorname{lin}}(k) . }
\end{aligned}
$$

In the case of cross-correlation between a gravitational tracer and a signal from DM annihilation, the matter power spectrum $P_{\delta \delta^{2}}$ involves terms of the type:

$$
\begin{aligned}
& \left\langle\sum_{i} \delta^{3}\left(\boldsymbol{x}_{1}-\boldsymbol{x}_{i}\right) \delta\left(m_{1}-m_{i}\right) \sum_{j} \delta^{3}\left(\boldsymbol{x}_{2}-\boldsymbol{x}_{j}\right) \delta\left(m_{2}-m_{j}\right)\right. \\
& \left.\sum_{k} \delta^{3}\left(\boldsymbol{x}_{3}-\boldsymbol{x}_{k}\right) \delta\left(m_{3}-m_{k}\right)\right\rangle
\end{aligned}
$$

For the same reasons discussed above in connection with the auto-correlation of annihilating DM, only the terms in the r.h.s. of Equation (8) survive, leading again to Equation (9) for the two-point correlation function (see also Appendix B in Ref. [4]). This allows to write the one- and two-halo terms of the PS of cross-correlation with lensing or decaying DM as:

$$
\begin{aligned}
P_{\delta \delta^{2}}^{1 h}(k)= & \int_{m_{\min }}^{m_{\max }} d m \frac{d n}{d m} \tilde{v}(k \mid m) \frac{\tilde{u}(k \mid m)}{\Delta^{2}} \\
P_{\delta \delta^{2}}^{2 h}(k)= & {\left[\int_{m_{\min }}^{m_{\max }} d m \frac{d n}{d m} b_{h}(m) \tilde{v}(k \mid m)\right] } \\
& {\left[\int_{m_{\min }}^{m_{\max }} d m \frac{d n}{d m} b_{h}(m) \frac{\tilde{u}(k \mid m)}{\Delta^{2}}\right] P^{\operatorname{lin}}(k), }
\end{aligned}
$$

The PS in the case of cross-correlation of annihilating DM with astrophysical sources is:

$$
\begin{aligned}
P_{S \delta^{2}}^{1 h}(k, z)= & \int_{\mathcal{L}_{\min }(z)}^{\mathcal{L}_{\max }(z)} d \mathcal{L} \Phi(\mathcal{L}, z) \frac{\mathcal{L}}{\left\langle g_{S}\right\rangle} \frac{\tilde{u}(k \mid m(\mathcal{L}))}{\Delta^{2}} \\
P_{S \delta^{2}}^{2 h}(k, z)= & {\left[\int_{\mathcal{L}_{\min }(z)}^{\mathcal{L}_{\max }(z)} d \mathcal{L} \Phi(\mathcal{L}, z) b_{S}(\mathcal{L}, z) \frac{\mathcal{L}}{\left\langle g_{S}\right\rangle}\right] } \\
& {\left[\int_{m_{\min }}^{m_{\max }} d m \frac{d n}{d m} b_{h}(m) \frac{\tilde{u}(k \mid m)}{\Delta^{2}}\right] P^{\operatorname{lin}}(k, z), }
\end{aligned}
$$

and in the approximate scenario is:

$$
\begin{aligned}
P_{\text {gal }, \delta^{2}}^{1 h}(k)= & \int_{m_{\min }}^{m_{\max }} d m \frac{d n}{d m} \frac{\left\langle N_{\text {gal }}\right\rangle}{\bar{n}_{\text {gal }}} \tilde{v}(k \mid m) \frac{\tilde{u}(k \mid m)}{\Delta^{2}} \\
P_{\text {gal }, \delta^{2}}^{2 h}(k)= & {\left[\int_{m_{\min }}^{m_{\max }} d m \frac{d n}{d m} b_{h}(m) \frac{\left\langle N_{\text {gal }}\right\rangle}{\bar{n}_{\text {gal }}} \tilde{v}(k \mid m)\right] } \\
& {\left[\int_{m_{\text {min }}}^{m_{\text {max }}} d m \frac{d n}{d m} b_{h}(m) \frac{\tilde{u}(k \mid m)}{\Delta^{2}}\right] P^{\text {lin }}(k), }
\end{aligned}
$$


A few examples of power spectra for the different crosscorrelation cases are shown in Figure 2, for both annihilating and decaying DM with lensing or galaxy tracers.

\section{WINDOW FUNCTIONS}

The final step is to determine the window functions of the different observables. Let us start with the gravitational tracers. The window function of the lensing signal takes the form (see, e.g., Ref. [30]):

$$
W(\chi)=\frac{3}{2} H_{0}^{2} \Omega_{\mathrm{m}}[1+z(\chi)] \chi \int_{\chi}^{\infty} \mathrm{d} \chi^{\prime} \frac{\chi^{\prime}-\chi}{\chi^{\prime}} \frac{\mathrm{d} N}{\mathrm{~d} \chi^{\prime}}\left(\chi^{\prime}\right)
$$

where $H_{0}$ is the Hubble constant, $\Omega_{\mathrm{m}}$ the matter-density parameter and $\mathrm{d} N / \mathrm{d} \chi$ denotes the redshift distribution of the background sources (which are lensed by the structures), normalized to unity area.

In the case of $\mathrm{CMB}$ lensing, the source is provided by the last scattering surface. Approximating it as a surface with infinitesimal width located at $z_{*}$, we can use the same Equation (33) with $\mathrm{d} N / \mathrm{d} z=\delta\left(z-z_{*}\right)$. In this case we obtain:

$$
W(\chi)=\frac{3}{2} H_{0}^{2} \Omega_{\mathrm{m}}[1+z(\chi)] \chi \frac{\chi_{*}-\chi}{\chi_{*}}
$$

In the case of astrophysical sources, as e.g., the LSS tracers, we have (see, e.g., Ref. [16]):

$$
W(E, z)=\frac{\left\langle g_{S}(z)\right\rangle}{4 \pi(1+z)} e^{-\tau[E(1+z), z]} .
$$

However, in this paper, following the discussion outlined in the previous section, we employ a common description for all types of galaxies with a window function given by $W=d n_{\text {gal }} / d z$.

For the case of decaying DM we again have $f(\boldsymbol{x})=\delta(\boldsymbol{x})$ and the window function is given by (see, e.g., Ref. [6]):

$$
W(E, z)=\frac{1}{4 \pi} \frac{\Omega_{\mathrm{DM}} \rho_{c}}{m_{\chi} \tau_{d}} \frac{d N_{d}[E(1+z)]}{d E} e^{-\tau[E(1+z), z]}
$$

where $\Omega_{\mathrm{DM}}$ is the DM density parameter, $\rho_{c}$ is the critical density of the Universe, $m_{\chi}$ and $\tau_{d}$ denote the mass and decay lifetime of the DM particle, respectively, and $d N_{d}[E] / d E$ is the number of photons (at radio, $\mathrm{X}$-ray, or $\gamma$-ray frequency, depending in the specific signal under study) emitted per decay-event in the energy range $(E, E+d E)$. Finally, $\tau$ is the optical depth for absorption. It can be relevant in the gamma-ray case and is mainly due to pair production on the extragalactic background light emitted by galaxies in the ultraviolet, optical, and infrared bands. For radio and X-rays, the absorption can have an impact only in the very low frequency part of the bands. Notice that the factor $\Omega_{\mathrm{DM}} \rho_{c}$ comes from the normalization of $W$, such that $\left\langle g_{d}\right\rangle=\bar{\rho}$. Notice also that we are considering the differential energy flux as the intensity $I$ under study: thus we quote differential (in energy) window functions for the DM electromagnetic signals.

In the case of annihilating DM, the signal scales with $\rho^{2}$, and to define the corresponding window function we can make use of the so-called clumping factor $\Delta^{2}(z)$, defined as:

$$
\Delta^{2}(z)=\frac{\left\langle\rho^{2}\right\rangle}{\bar{\rho}^{2}}=\int_{m_{\min }}^{m_{\max }} d m \frac{d n}{d m} \int d^{3} \boldsymbol{x} \frac{\rho^{2}(\boldsymbol{x} \mid m)}{\bar{\rho}^{2}},
$$

The window function for annihilating DM then takes the form (see, e.g., Ref. [3]):

$$
\begin{aligned}
W(E, z)= & \frac{\left(\Omega_{\mathrm{DM}} \rho_{c}\right)^{2}}{4 \pi} \frac{\left\langle\sigma_{a} v\right\rangle}{2 m_{\chi}^{2}}(1+z)^{3} \\
& \Delta^{2}(z) \frac{d N_{a}[E(1+z)]}{d E} e^{-\tau[E(1+z), z]},
\end{aligned}
$$

where $\left\langle\sigma_{a} v\right\rangle$ is the velocity-averaged annihilation cross sections times relative velocity (which we assume to be the same in all DM structures) and $d N_{a}[E] / d E$ is the number of photons emitted per annihilation event in the energy range $(E, E+d E)$.

\section{DISCUSSION}

Let us turn now to the discussion of some examples of auto- and cross-correlation angular power spectra. To be definite, and for illustrative purposes, we focus on a benchmark DM candidate with mass $m_{\chi}=100 \mathrm{GeV}$, and final state of annihilation/decay into quarks $b-\bar{b}$. Since we will report dimensionless window functions and angular power spectra (i.e., quantities relative to the total average intensity), the actual values of the annihilation cross section or the decay lifetime are not relevant in the discussion. For definiteness, the substructure description is chosen to follow the VC scenario mentioned above.

Concerning LSS tracers, we will consider two specific examples, given by the population of galaxies observed by the 2MASS [31] and NVSS [32] surveys. The parameters entering in the window function $d n_{\text {gal }} / d z$ of the two cases are taken from Refs. $[33,34]$.

In the case of weak-lensing shear, we consider the distributions of background sources that will be relevant for the Dark Energy Survey (DES) telescope and for the Euclid satellite. The source distribution for DES [35] can be described by $d N / \mathrm{d} z=A_{D}\left(z^{a}+\right.$ $\left.z^{a b}\right) /\left(z^{b}+c\right)$, with $a, b$, and $c$ provided in Table 1 of Ref. [36], and $A_{D}$ fixed by the normalization condition $\int d z \mathrm{dN} / \mathrm{d} z=1$. For Euclid [37, 38], we can use $d N / \mathrm{d} z=A_{E} z^{2} \exp \left[-\left(z / z_{0}\right)^{1.5}\right]$, where $z_{0}=z_{m} / 1.4$ with $z_{m}=0.9$ being the median redshift of the survey and $A_{E}$ again fixed by the normalization.

In the right panel of Figure 2 we show the cross-correlation 3D PS of annihilating/decaying DM with lensing and LSS tracers, computed as described in the previous sections. Notice that the PS for the cross-correlation between lensing with decaying $\mathrm{DM}$ is the customary matter power spectrum $P_{\delta \delta}$ (described in Equations 14, 15). The other cases (LSS with decaying DM and the cases with annihilating DM) lie above it, having more power on the structure scales $k \gtrsim 1 \mathrm{Mpc}^{-1}$. This is particularly true in the annihilating case and can be also understood by comparing Figure 1 with the left panel of Figure 2 in the VC scheme.

The window functions are shown in Figure 3. The left panel refers to the particle DM cases and shows the redshift dependence of the window functions for the radio, X-ray, and gamma-ray emissions, in both annihilating and decaying scenarios. The radio 


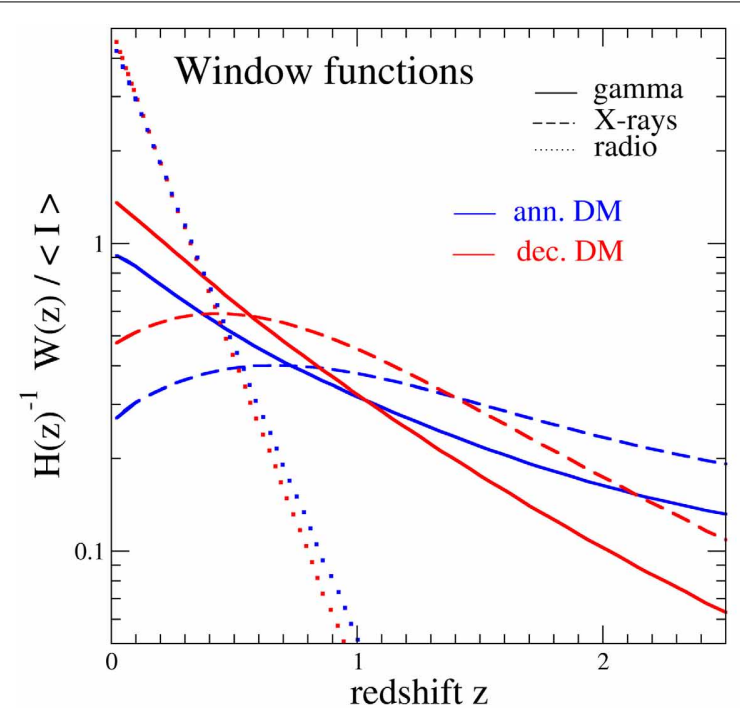

FIGURE 3 | Window functions. Left: Window functions as a function of the redshift for radio, X-ray, and gamma-ray emissions from annihilating/decaying DM. The window functions refer to reference frequencies/energies: $v=1$ $\mathrm{GHz}$ for radio, $E=10 \mathrm{keV}$ for $\mathrm{X}$-rays, $E=1 \mathrm{GeV}$ for gamma-rays. They have

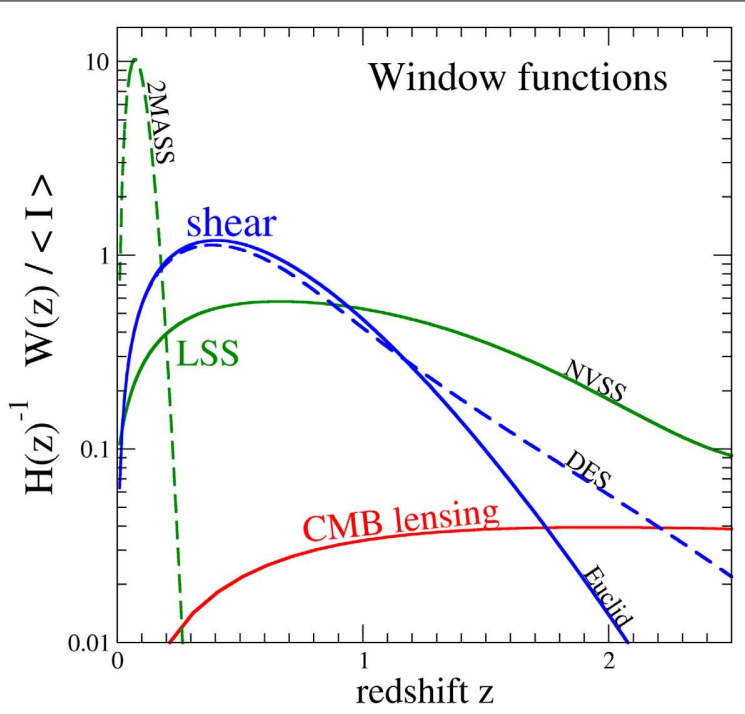

been normalized to the total average intensity $\langle I\rangle$ such that the integral of the curves is equal to 1 for all cases. Right: Window functions vs. redshift for cosmic shear, CMB lensing, and two populations of galaxies (detected in the 2MASS and NVSS surveys) tracing LSS. signal is due to synchrotron-emission produced by electrons and positrons (induced by the DM annihilations/decays) interacting with a magnetic field chosen to be constant and with strength $B=$ $10 \mu \mathrm{G}$. The X-ray flux instead arises from inverse Compton (IC) of the same electrons and positrons on the $\mathrm{CMB}$, with ensuing up-scatter of the CMB photons. The window functions depend on the photon energy, as is clear from Equations $(36,39)$ : for definiteness, we choose some reference frequency/energy, such that the different emissions are all involving $\mathrm{GeV}$ particles (photons in the gamma-ray case, while $e^{+} / e^{-}$in the radio and X-ray case) originated from the annihilations/decays. The reference frequencies are: $\nu=1 \mathrm{GHz}$ for radio, $E=10 \mathrm{keV}$ for X-rays, $E=$ $1 \mathrm{GeV}$ for gamma-rays. The plot shows that a multi-wavelength approach can be quite powerful. Indeed, the different emissions exhibit rather different window functions: each one therefore can provide different and complementary information. In particular, the radio case is more peaked at low redshift, while the X-ray window function has a flatter shape, with the gamma-ray case being somewhat in between. This is due to the fact that the CMB becomes much more dense in the past (the photon energy-density goes with the well known $(1+z)^{4}$ scaling), making the energy losses for IC very effective at higher redshifts: this depletes the radio signal at high $z$, while, in the X-ray case, the corresponding increase of the IC emissivity compensates the effect. We also notice that the decaying and annihilating DM cases produce very similar window functions for radio emission, while for X-rays and gamma-rays the window function is flatter in the annihilating case as compared to the decaying case. This occurs because of the effect of clustering, which enhances the signal at larger redshifts.

The window functions for weak-lensing shear (DES and EUCLID populations), CMB lensing, and LSS (2MASS and NVSS galaxy catalogs) are shown in the right panel of Figure 3. They have quite different shapes, as a consequence of the different redshift evolution of the relevant tracers which produce the observed signal. Since the cross-correlation power spectrum depends on the overlap of the window functions of the two correlated signals, as is shown in Equation (5), the different gravitational tracers will be more/less efficient in the cross-correlation with particle DM signals. Comparing the two panels of Figure 3 allows to understand which cross-correlation combinations could be optimal (although experimental limitations may occur, like limited statistics in one observational channel or angular resolutions). Notice that 2MASS and NVSS are sort of two opposite limits for galaxy tracers (and this is the why we choose these examples): 2MASS coverage peaks at very low-redshifts, below 0.2 , while NVSS has a broad redshift distribution. The weak-lensing shear is peaked at intermediate-low redshift, which makes this observables a good candidate for cross-correlation with particle DM signals, as proposed in Ref. [16]. The CMB window function instead extends over a very wide range of redshift (in principle, up to the last scattering): this is also why it appears significantly lower, as compared to the other cases in Figure 3, since we have normalized all window functions to the total average intensity (i.e., the curves shown in Figure 3 are normalized to 1). In the following, we also normalize all the angular PS to the total averaged intensity, as reported in Equation (5), in order to make the various cases more easily comparable. The results will therefore show the fractional size of the anisotropy signal [multiplied by $\ell(\ell+1) /(2 \pi)$ ] with respect to the average isotropic intensity field.

The angular power spectra of annihilating DM are presented in the left panels of Figures 4-6, while the decaying cases are reported in the right panels of the same figures. Figure 4 shows the auto- and cross-correlations of the electromagnetic signals. The most anisotropic cases involve the radio emission. This is 


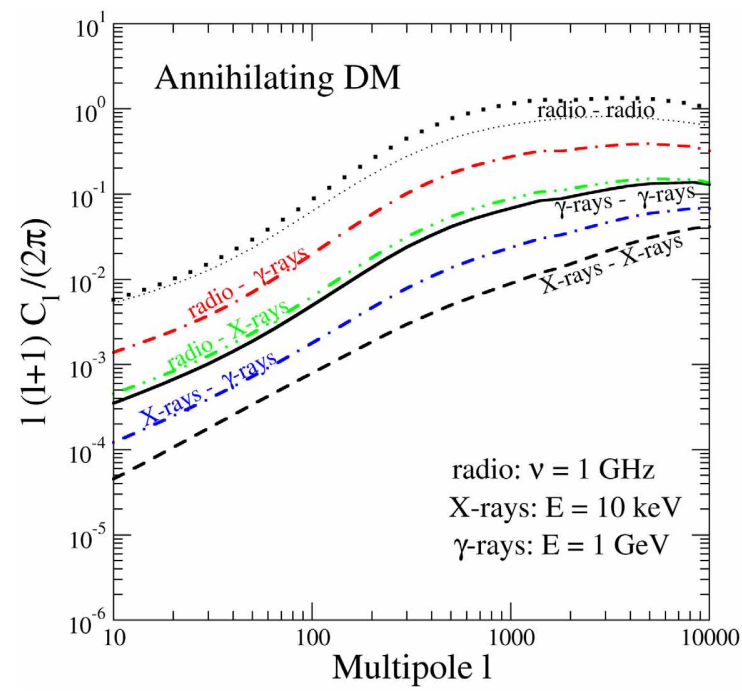

FIGURE 4 | Angular PS of radio, X-ray, and gamma-ray emissions (autoand cross-correlations) from annihilating DM (left panel) and decaying DM (right panel). The computation is performed using the 3D PS models reported

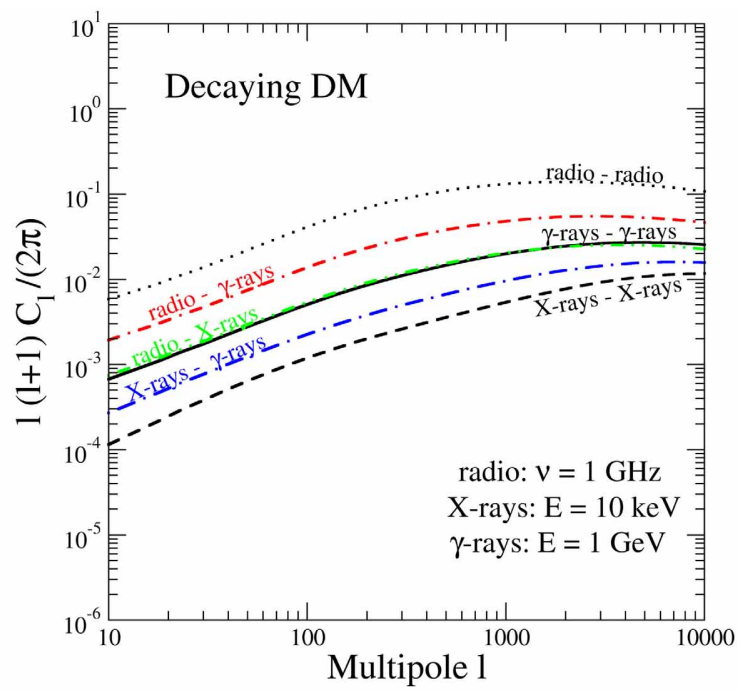

in Figure $\mathbf{1}$ and the window functions shown in Figure 3. For the radio-radio case, we also show (with the thinner dotted line) the effect of a cored DM profile replacing the NFW distribution (as a possible results of particle diffusion).

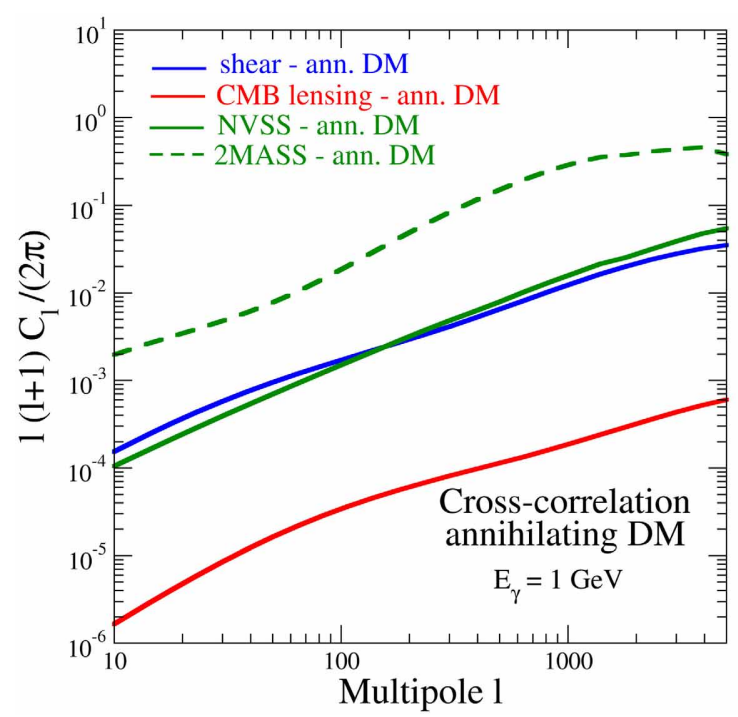

FIGURE 5 |Angular cross-correlation PS between gamma-rays (at $E_{y}=1 \mathrm{GeV}$ ) and gravitational tracers (cosmic shear, CMB lensing and LSS tracers), for annihilating DM (left panel) and

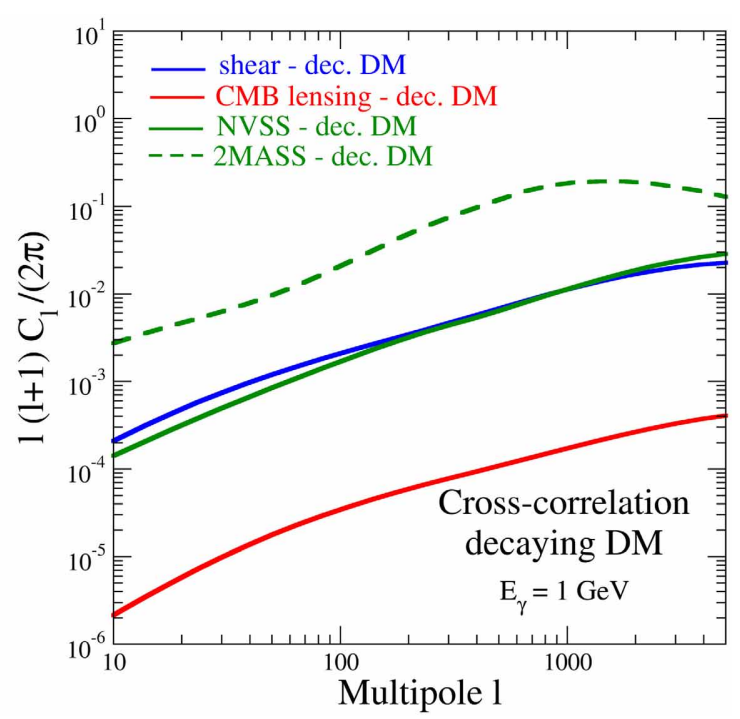

decaying DM (right panel). The computation is performed using the 3D PS models reported in Figure $\mathbf{2}$ and the window functions shown in Figure $\mathbf{3}$. because the radio window function is peaked at low-redshift: the signal therefore comes from a relatively low number of halos and thus is quite anisotropic. The flatter window function of X-rays implies that in this case the number of objects contributing to the intensity field is larger, thus making the emission smoother and the ensuing PS smaller. The gamma-rays case is intermediate to radio and X-rays: as shown by its window function in Figure 3, the gamma-rays emission is peaked at low-to-intermediate redshifts and this implies a relatively larger anisotropy in the intensity, as compared to X-rays. We also notice that the radio PS flattens more rapidly at $\ell>10^{3}$ with respect to gamma and Xray PS. The 1-halo term takes over at large multipoles and leads to constant $C_{\ell}$ (Poisson-noise) when sources are point-like, while instead it leads to a decreasing $C_{\ell}$ when structures start to be resolved. The flattening is therefore again related to the redshift distribution. Indeed the inner structure of closer objects, which are more important in the radio case, is resolved at larger angular scale with respect to more distant objects which contribute to the bulk of the emission in the gamma and X-ray cases. We finally notice that at small angular scales, annihilating DM provides 


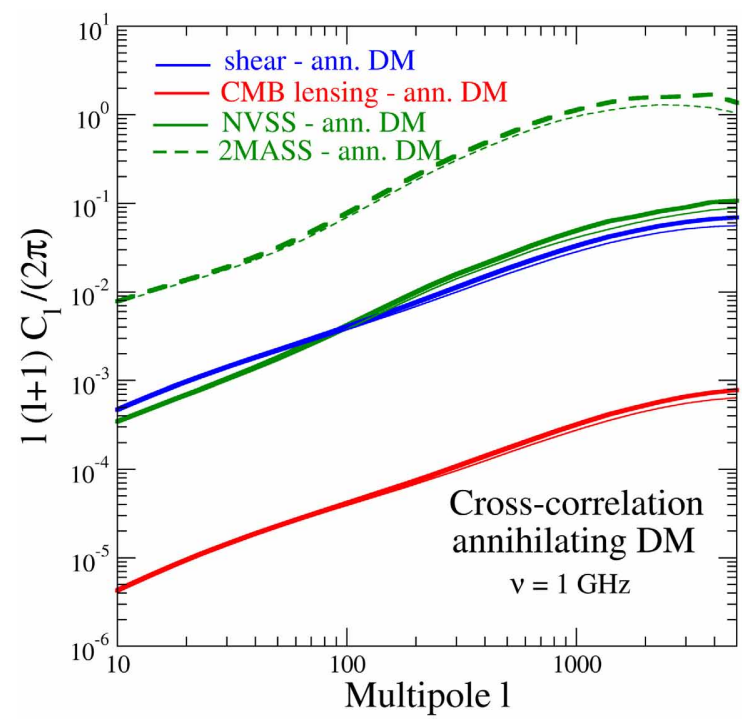

FIGURE 6 | Angular cross-correlation PS between the radio signals (at $v=1 \mathrm{GHz}$ ) and gravitational tracers (cosmic shear, CMB lensing and LSS tracers), for annihilating DM (left panel) and decaying DM (right panel). The computation is performed using the

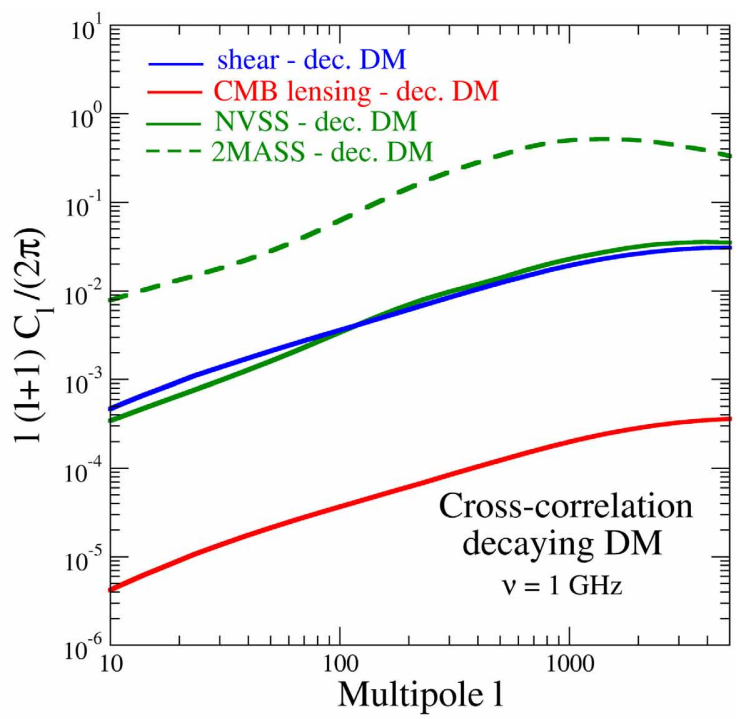

3D PS models reported in Figure $\mathbf{2}$ and the window functions shown in Figure 3. In the left panel, we also show (with thinner lines) the case of a cored DM distribution (as a possible results of particle diffusion). much more anisotropy as compared to decaying DM, due to its dependence on $\rho^{2}$ and to the subhalo scheme adopted.

As mentioned above, we are taking an approximate description for radiative emissions (both IC and synchrotron) where all the power is radiated at the same place of $e^{+} / e^{-}$injection, and therefore the density field of the emission is proportional to the DM density (for decaying DM) or DM density squared (for annihilating DM). One of the neglected effects is the diffusion of $e^{+} / e^{-}$, which might lead to a smoothing of the cuspy behavior of the density profile into a more cored profile (for a more extended discussion on the impact of this approximation on radio anisotropies, see Ref. [14]). If we still assume that all the power is radiated within the object, we can model the effect of diffusion on the final $e^{+} / e^{-}$distribution by replacing the NFW profile $\rho_{\mathrm{NFW}} \propto x^{-1}(1+x)^{-2}$ (where $x=r / r_{s}$ and $r_{s}$ is the scale radius) with a cored density, like e.g., $\rho_{\text {cored }} \propto(1+x)^{-1}\left(1+x^{2}\right)^{-1}$. The thick and thin dotted lines in the left panel of Figure 4 show the case with an NFW and cored distribution, respectively. The difference is not dramatic (notice that this can also be seen as an estimate for the case where the DM distribution itself is cored, although the power spectrum we have been using is based on results of $\mathrm{N}$-body simulations, thus fully consistent only with a cuspy profile).

Summarizing the behavior of the auto- and cross-correlation power spectra of the electromagnetic signals among themselves, we can state that the radio emission exhibits the strongest anisotropy, both among the auto-correlation signals and in combination with the other emissions. There are almost two orders of magnitude between the radio/radio auto-correlation signal as compared to the $\mathrm{X}$-rays/X-rays auto-correlation (which exhibit also the smallest among all the correlations of electromagnetic signals) and one order of magnitude larger than the gamma/gamma autocorrelation. Interesting prospects are present for the cross-correlation radio/gamma, which is a factor of 3-5 larger than the gamma/gamma case. These general features occur both for the annihilating and decaying DM signals, with more power at large multipoles for annihilating DM as compared to the decaying case, and with slightly more separation among the different cases again for annihilating DM as compared to decaying DM.

Since we are dealing here with angular power spectra normalized to the average intensity, the actual feasibility of detection will depend also on the absolute normalization level accessible by the different detectors, which is detector specific. Being concerned in this paper with the theoretical properties of the autoand cross-correlation signals and with the assessment of their mutual impact, we are not adopting here any specific experimental figure of merit: results shown in Figures 4-6 can then be folded with the individual detector capabilities. In fact, the experimental ability to disentangle an anisotropy signal will also depend on the specific features of the detectors and on the astrophysical backgrounds, which also produce an anisotropic electromagnetic emission. Photon detectors at different wavelengths have intrinsically different angular resolution: e.g., radio telescopes can resolve very fine details, and have an angular resolution which is much better than gamma-rays detectors. They will therefore be a suitable instrument for studying large multipoles $\ell$. On the other hand, at lower multipoles gamma-rays detector may be more suitable than interferometric radio telescopes. The combination of the information coming from the auto-correlation signal at different wavelengths, as well as cross-correlations of different signals, may therefore be a relevant tool to identify and characterize a DM signal. Concerning astrophysical backgrounds, a large number of electromagnetic emitters are present, like e.g., active galactic 
nuclei, star forming galaxies, etc.: their emission is anisotropic as well. The physical location of these astrophysical sources is to some extent correlated to the DM structures: nevertheless, they possess different properties both in redshift distribution (relevant for the ensuing window functions) and in spectral features. These differences can be potentially used to attempt a separation between these backgrounds and the DM signals discussed in this paper. Examples of the impact of astrophysical background have discussed in the case of the gamma-rays auto-correlation $[4,9-11,39,40]$, of the radio auto-correlation $[13,14]$ and of the cross-correlations gamma/shear [16]. In this paper we are concerned on assessing the size and the relative impact of DM signals. A detailed analysis of the background sources is beyond the scope of the present paper, and for the cases for which studies are present we refer to the quoted references.

The cross-correlation of the gamma and radio emissions with gravitational tracers is shown in Figures 5, 6, respectively. For definiteness, also here we have considered the gamma-rays emission at $E=1 \mathrm{GeV}$ and the radio emission art $\nu=1 \mathrm{GHz}$. We notice that the strongest correlation occurs with the 2MASS population, while the lowest is with the CMB lensing. This can be again understood by looking at the redshift dependence of the window functions. To have a good overlapping with the electromagnetic DM source, the gravitational tracer has to be peaked at relatively low redshift. Moreover, as we already discussed, the closer is the emission the more anisotropic it appears (and the ensuing angular PS flattens sooner, as a function of the multiple $l$ ). From the window function behavior of Figure 3, we see that the radio signal and the 2MASS tracer are both strongly peaked at very low redshifts: this fact enhances the cross-correlation both because of the large overlap and because closer sources are fewer and therefore more anisotropic. This is clearly seen in the cross-correlations results of Figure 6, where the angular PS for radio/2MASS is significantly large. In the left panel of Figure 6 we show again the effect of a cored distribution, which is even milder than for the auto-correlation PS (this occurs because this effect does not affect the LSS tracers and so now modifies only one of the two fields entering in the PS computation). Also the gamma/2MASS cross-correlation is relatively large, although smaller than the for the radio case. This is again due to the good overlap of the gamma-rays and 2MASS window functions, nevertheless to a lower extent than in the radio case.

The cross-correlations with cosmic shear (originally proposed for gamma/shear in Ref. [16]) and with NVSS have very similar angular PS, which could potentially offer a tool to disentangle a DM signal from astrophysical backgrounds. In the case of crosscorrelation with the cosmic-shear, a tomographic approach is feasible, and represents an unique opportunity to test the different redshift scaling we have been discussing (Camera et al., submitted).

The cross-correlation with the CBM lensing observable is the smallest, due to the fact that CMB-lensing sources are distributed in a much deeper interval of redshift, as compared to the electromagnetic emitters. We finally notice that the decaying DM cross-correlation angular PS (but this applies also for the autocorrelation case), even though they have a behavior similar to the annihilating case, nevertheless exhibit a slower growth of $\ell(\ell+1) C_{\ell}$, which means less power at small scales: this can be understood by comparing the annihilating and decaying $3 \mathrm{D}$ power spectra in Figure 2 at $k \gtrsim 1 \mathrm{Mpc}^{-1}$.

Summarizing the behavior of the cross-correlation power spectra of the electromagnetic signals with the gravitational tracers, we can state that again the radio emission exhibits the strongest anisotropy signal. The largest effect occurs (both for radio and gamma-rays) in the cross-correlation to the lowredshift 2MASS population: the angular PS spectrum in this case is about one order of magnitude stronger than the crosscorrelations with cosmic shear and NVSS, and about three orders of magnitude larger than the cross-correlation with the CMB-lensing observable. We caution again that, however, such differences do not straightforwardly translate into the actual experimental capabilities.

\section{CONCLUSIONS}

In this paper we have discussed extragalactic anisotropies in the electromagnetic emission produced by DM annihilation or decay as a promising tool to search for a DM signal. We have first reviewed the formalism needed to compute a generic 2-point angular power spectrum by following the halo-model description of clustering of structures in the Universe. This formalism was then applied to realize estimates involving relevant emissions induced by particle DM annihilations or decays. We have discussed the features and the relative size of the various auto- and cross-correlation angular power spectra that can be envisaged for anisotropy studies.

From the side of DM signals we have considered the full multi-wavelength spectrum, including the synchrotron emission at radio frequencies, the IC radiation in the X-ray and gamma-ray bands, as well as the prompt emission of gamma-rays. The angular power spectra of auto-correlation of each of these signals and of the cross-correlation between any pair of them is presented.

As a way to enhance the capability of detection of such nongravitational signals of DM (and to improve their disentanglement from other astrophysical backgrounds) we introduce their cross-correlation with maps tracing the gravitational potential. We have analyzed this possibility studying specific gravitational tracers of DM distribution in the Universe: weak-lensing cosmic shear, LSS matter distribution and CMB-lensing.

We have shown that cross-correlating a multi-wavelength DM signal (which is a direct manifestation of its particle physics nature) with a gravitational tracer (which is a manifestation of the presence of large amounts of unseen matter in the Universe) may offer a prime tool to demonstrate that what we call DM is indeed formed by an elementary particle.

\section{ACKNOWLEDGMENTS}

Work supported by the INFN research grant Astroparticle Physics Project-FA51 and by the Strategic Research Grant: Origin and Detection of Galactic and Extragalactic Cosmic Rays funded by Torino University and Compagnia di San Paolo. Nicolao Fornengo acknowledges support of the spanish MICINN Consolider Ingenio 2010 Programme MULTIDARK CSD2009-00064. 


\section{REFERENCES}

1. Ade PAR, Aghanim N, Armitage-Caplan C, Arnaud M, Ashdown M, Atrio-Barandela F, et al. [Planck Collaboration]. Planck 2013 results. XVI. Cosmological parameters. arXiv:1303.5076 [astro-ph.CO].

2. Bertone G, Silk J, Moore B, Diemand J, Bullock J, Kaplinghat M, et al. Particle Dark Matter: Observations, Models and Searches. Cambridge, UK: Cambridge University Press (2010), p. 738. doi: 10.1017/CBO9780511770739

3. Ando S, Komatsu E. Anisotropy of the cosmic gamma-ray background from dark matter annihilation. Phys Rev D. (2006) 73:023521. doi: 10.1103/PhysRevD.73.023521

4. Ando S, Komatsu E, Narumoto T, Totani T. Dark matter annihilation or unresolved astrophysical sources? Anisotropy probe of the origin of cosmic gamma-ray background. Phys Rev D. (2007) 75:063519. doi: 10.1103/PhysRevD.75.063519

5. Fornasa M, Pieri L, Bertone G, Branchini E. Anisotropy probe of galactic and extra-galactic dark matter annihilations. Phys Rev D. (2009) 80:023518. arXiv:0901.2921 [astro-ph.CO].

6. Ibarra A, Tran D, Weniger C. Detecting gamma-ray anisotropies from decaying dark matter: prospects for Fermi LAT. Phys Rev D. (2010) 81:023529. arXiv:0909.3514 [hep-ph].

7. Cuoco A, Sellerholm A, Conrad J, Hannestad S. Anisotropies in the diffuse gamma-ray background from dark matter with Fermi LAT: a closer look. Mon Not Roy Astron Soc. (2011) 414:2040. arXiv:1005.0843 [astro-ph.HE].

8. Fornasa M, Zavala J, Sanchez-Conde MA, Siegal-Gaskins JM, Delahaye T, Prada F, et al. Characterization of dark-matter-induced anisotropies in the diffuse gamma-ray background. MNRAS (2013) 1529:429. arXiv:1207.0502 [astro-ph.HE].

9. Ripken J, Cuoco A, Zechlin H-S, Conrad J, Horns D. The sensitivity of Cherenkov telescopes to dark matter and astrophysically induced anisotropies in the diffuse gamma-ray background (2012). arXiv:1211.6922 [astro-ph.HE].

10. Hensley BS, Pavlidou V, Siegal-Gaskins JM. Novel techniques for decomposing diffuse backgrounds. MNRAS. (2013) 591:433. arXiv:1210.7239 [astroph.CO].

11. Ando S, Komatsu E. Constraints on the annihilation cross section of dark matter particles from anisotropies in the diffuse gamma-ray background measured with Fermi-LAT. Phys Rev D. (2013) 87:123539. arXiv:1301.5901 [astro-ph.CO].

12. Ackermann M, Ajello M, Albert A, Baldini L, Ballet J, Barbiellini G, et al. [(Fermi LAT Collaboration]. Anisotropies in the diffuse gamma-ray background measured by the Fermi LAT. Phys Rev D. (2012) 85:083007. doi: 10.1103/PhysRevD.85.083007

13. Zhang L, Sigl G. Dark matter signatures in the anisotropic radio sky. JCAP (2008) 0809:027. arXiv:0807.3429 [astro-ph].

14. Fornengo N, Lineros R, Regis M, Taoso M. Cosmological radio emission induced by WIMP dark matter. JCAP (2012) 1203:033. arXiv:1112.4517 [astro-ph.CO].

15. Inoue Y, Murase K, Madejski GM, Uchiyama Y. Probing the cosmic X-ray and $\mathrm{MeV}$ gamma-ray background radiation through the anisotropy. Astrophys J. (2013) 776:33. arXiv:1308.1951 [astro-ph.CO].

16. Camera S, Fornasa M, Fornengo N, Regis M. A novel approach in the weakly interacting massive particle quest: cross-correlation of gamma-ray anisotropies and cosmic shear. Astrophys J. (2013) 771:L5. arXiv:1212.5018 [astro-ph.CO].

17. Ando S, Benoit-Levy AEL, Komatsu E. Mapping dark matter in the gamma-ray sky with galaxy catalogs. (Just when this paper was submitted, an independent analysis discussing cross-correlation of gamma-rays produced by dark matter annihilation with galaxy catalogs appeared). arXiv:1312.4403 [astro-ph.CO].

18. Limber DN. The analysis of counts of the extragalactic nebulae in terms of a fluctuating density field. Astrophys J. (1953) 117:134L. doi: 10.1086/145672

19. Limber DN, Kaiser N. Weak gravitational lensing of distant galaxies Astrophys J. (1992) 388:272. doi: 10.1086/171151

20. Limber DN, Kaiser N. Weak lensing and cosmology. Astrophys J. (1998) 498:26. doi: $10.1086 / 305515$

21. Scherrer R, Bertschinger E. Statistics of primordial density perturbations from discrete seed masses. Astrophys J. (1991) 381:349. doi: 10.1086/170658

22. Sheth RK, Tormen G. Large scale bias and the peak background split. Mon Not Roy Astron Soc. (1999) 308:119. arXiv:astro-ph/9901122.
23. Munoz-Cuartas JC, Maccio AV, Gottlober S, Dutton AA. The redshift evolution of LCDM halo parameters: concentration, spin, and shape. Mon Not Roy Astron Soc (2011) 411:584. arXiv:1007.0438 [astro-ph.CO].

24. Bullock JS, Kolatt TS, Sigad Y, Somerville RS, Kravtsov AV, Klypin AA, et al. Profiles of dark haloes. Evolution, scatter, and environment. Mon Not Roy Astron Soc. (2001) 321:559-75. astro-ph/9908159.

25. Navarro JF, Frenk CS, White SDM. A universal density profile from hierarchical clustering. Astrophys J. (1997) 490:493. arXiv:astro-ph/9611107.

26. Cooray A, Sheth RK. Halo models of large scale structure. Phys Rept. (2002) 372:1. astro-ph/0206508.

27. Takahashi R, Sato M, Nishimichi T, Taruya A, Oguri M. Revising the halofit model for the nonlinear matter power spectrum. Astrophys J. (2012) 761:152. arXiv:1208.2701 [astro-ph.CO].

28. Zheng Z, Berlind AA, Weinberg DH, Benson AJ, Baugh CM, Cole S, et al. Theoretical models of the halo occupation distribution: separating central and satellite galaxies. Astrophys J. (2005) 633:791. astro-ph/0408564.

29. Kamionkowski M, Koushiappas SM, Kuhlen M. Galactic substructure and dark matter annihilation in the milky way halo. Phys Rev D. (2010) 81:043532. arXiv:1001.3144 [astro-ph.GA].

30. Bartelmann M. Gravitational lensing. Class Quant Grav. (2010) 27:233011. doi: 10.1088/0264-9381/27/23/233001

31. Skrutskie MF, Cutri RM, Stiening R, Weinberg MD, Schneider S, Carpenter JM. The two micron all sky survey (2MASS). Astron J. (2006) 131:1163. doi: $10.1086 / 498708$

32. Condon JJ, Cotton WD, Greisen EW, Yin QF, Perley RA, Taylor GB, et al. The NRAO VLA sky survey. Astron J. (1998) 115 :1693-1716. doi: 10.1086/300337

33. Afshordi N, Loh Y-S, Strauss MA. Cross-correlation of the cosmic microwave background with the 2MASS galaxy survey: signatures of dark energy, hot gas, and point sources. Phys Rev D (2004) 69:083524. doi: 10.1103/PhysRevD.69.083524

34. De Zotti G, Massardi M, Negrello M, Wall J. Radio and millimeter continuum surveys and their astrophysical implications. Astron Astrophys Rev. (2010) 18:1-65. arXiv:0908.1896 [astro-ph.CO].

35. Abbott T, Aldering G, Annis J, Barlow M, Bebek C, Bigelow B. [The Dark Energy Survey Collaboration]. The dark energy survey. Available online at: http://arxiv.org/abs/astro-ph/0510346

36. Fu L, Semboloni E, Hoekstra H, Kilbinger M, van Waerbeke L, Tereno I. Very weak lensing in the CFHTLS Wide: cosmology from cosmic shear in the linear regime. Astron Astrophys. (2008) 479:9. arXiv:0712.0884 [astro-ph].

37. Laureijs R, Amiaux J, Arduini S, Augueres JL, Brinchmann J, Cole R. Euclid Definition Study Report (2011). arXiv:1110.3193 [astro-ph.CO].

38. Amendola L, Appleby S, Bacon D, Baker T, Baldi M, Bartolo N. [Euclid Theory Working Group Collab.]. Cosmology and fundamental physics with the Euclid satellite (2012). arXiv:1206.1225 [astro-ph.CO]. Available online at: http://www.euclid-ec.org

39. Taoso M, 'i. Ando S, Bertone G, Profumo S. Angular correlations in the cosmic gamma-ray background from dark matter annihilation around intermediate-mass black holes. Phys Rev D (2009) 79:043521 [arXiv:0811.4493 [astro-ph]].

40. Xia JQ, Cuoco A, Branchini E, Fornasa M, Viel M. A cross-correlation study of the Fermi-LAT $\gamma$-ray diffuse extragalactic signal. Mon Not Roy Astron Soc. (2011) 416:2247. arXiv:1103.4861 [astro-ph.CO]

Conflict of Interest Statement: The authors declare that the research was conducted in the absence of any commercial or financial relationships that could be construed as a potential conflict of interest.

Received: 17 December 2013; accepted: 27 January 2014; published online: 18 February 2014.

Citation: Fornengo $N$ and Regis $M$ (2014) Particle dark matter searches in the anisotropic sky. Front. Physics 2:6. doi: 10.3389/fphy.2014.00006

This article was submitted to High-Energy and Astroparticle Physics, a section of the journal Frontiers in Physics.

Copyright (C) 2014 Fornengo and Regis. This is an open-access article distributed under the terms of the Creative Commons Attribution License (CC BY). The use, distribution or reproduction in other forums is permitted, provided the original author(s) or licensor are credited and that the original publication in this journal is cited, in accordance with accepted academic practice. No use, distribution or reproduction is permitted which does not comply with these terms. 